\section{Junctional Escape and Respiratory Sinus Arrhythmia with Acute Cholecystitis}

\section{Abstract}

Background: Bradycardias have been reported (rarely) associated with acute cholecystitis.

Data extraction: We here present the first reported case in which acute cholecystitis was associated with self-limited episodes of slow junctional escape (40/min) and marked sinus arrhythmia.

Conclusion: Brady-arrhythmias have been associated with acute biliary disease consistent with the putative mechanism of the "cardiobiliary reflex", i.e., marked vagotonia.

Keywords: Cholecystitis; Bradycardia; Junctional escape; Respiratory sinus arrhythmia; Vagotonia

Received: July 12, 2016; Accepted: July 27, 2016; Published: August 03, 2016

\section{Constantine A Manthous \\ Lawrence \& Memorial Hospital, Internal Medicine and Critical Care Medicine, 6 Hemingway Road, Niantic, CT, USA}

\section{Corresponding author: \\ Constantine A Manthous}

\section{झ constantinemanthous@gmail.com}

Lawrence \& Memorial Hospital, Internal Medicine and Critical Care Medicine, 6 Hemingway Road, Niantic, CT, USA.

Citation: Manthous CA. Junctional Escape and Respiratory Sinus Arrhythmia with Acute Cholecystitis. J Intensive \& Crit Care 2016, 2:3.
While some widely scattered case reports [1-3] and reviews [4] have suggested a connection between acute biliary disease and abnormal electrocardiogram, the connection has remained largely theoretical. We here report the case of a patient with acute cholecystitis who presented with junctional escape and marked sinus arrhythmia consistent with excess vagotonia hypothesized to be the cause in one previous similar report and reviews [1-4].

\section{Case Report}

A 76 year old lady with a history of coronary artery disease, remote cerebral vascular accident, obstructive sleep apnea, gastroesophageal reflux disease and overactive bladder presented with $24 \mathrm{~h}$ of chills and nausea. Her medications included carvedilol, losartan, mirabegron, pantoprazole, aspirin, and clopidogrel. Her physical examination was remarkable for baseline sinus rhythm (in the 60's) but with recurring episodes of sinus arrest and abnormally slow junctional escape in the 40s/min (Figure 1). Her blood pressure remained above 90 $\mathrm{mmHg}$ even with severe bradycardia. Room air saturation was $88 \%$ and corrected with $2 \mathrm{~L}$ nasal cannula. Physical examination was unremarkable except for a $3 / 6$ systolic murmur. Abdomen was not initially tender on admission. She was mildly anemia (Hgb $10.2 \mathrm{~g} / \mathrm{dl}$ ) and thrombocytopenic $\left(118,000 / \mathrm{mm}^{3}\right)$ with admission white count of $4.0 \times 10^{3} / \mathrm{mm}^{3}$. Procalcitonin was markedly positive at $33.7 \mathrm{ng} / \mathrm{ml}$. Urinalysis was remarkable for pyuria and later grew $>10^{5} \mathrm{E}$. coli. Electrolytes were remarkable for a potassium $5.5 \mathrm{mmol} / \mathrm{L}$ of BUN of $40 \mathrm{mg} / \mathrm{dl}$ and creatinine of $1.4 \mathrm{mg} / \mathrm{dl}$. Labs also included a positive d-dimer with normal fibrinogen, total bilirubin $2.0 \mathrm{mg} / \mathrm{dl}$, SGOT $719 \mathrm{U} / \mathrm{L}$, SGPT $491 \mathrm{U} / \mathrm{L}$, alkaline phosphatase $401 \mathrm{U} / \mathrm{L}$. Troponin was initially normal and peaked at 24 hours at $0.16 \mathrm{ng} / \mathrm{ml}$ while CPK remained entirely normal. A temporary transvenous pacemaker was placed for the first $48 \mathrm{~h}$ until episodes of bradycardia abated. Right upper quadrant ultrasound showed gall bladder thickening with normal biliary ducts. Cholescintography showed a non-filling, hyperemic gall bladder with stones/sludge suggestive of cholecystitis. On reexamination on day \#2-3, she was noted to have some tenderness of her right upper quadrant and remarkable respiratory-phasic variability of heart rate; rhythm strip demonstrated marked sinus arrhythmia (Figure 2). She was deemed a high risk for surgery, and instead was treated with a long course of antibiotics. She went on to a full recovery, without pacemaker or further cardiovascular complications.

\section{Discussion}

To our knowledge, this is the $5^{\text {th }}$ reported case of acute biliary disease accompanied by bradycardia, and the first case demonstrating abnormally slow junctional escape and marked sinus arrhythmia. O'Reilly and Krauthamer reported two patients whose cholecystitis was accompanied by "regular" bradycardia $50-60 / \mathrm{min}$ in one and sinus bradycardia of $38-42 / \mathrm{min}$ in the other. Both resolved with cholecystectomy. Lau and colleagues described a patient with acalculous cholecystitis with sinus 


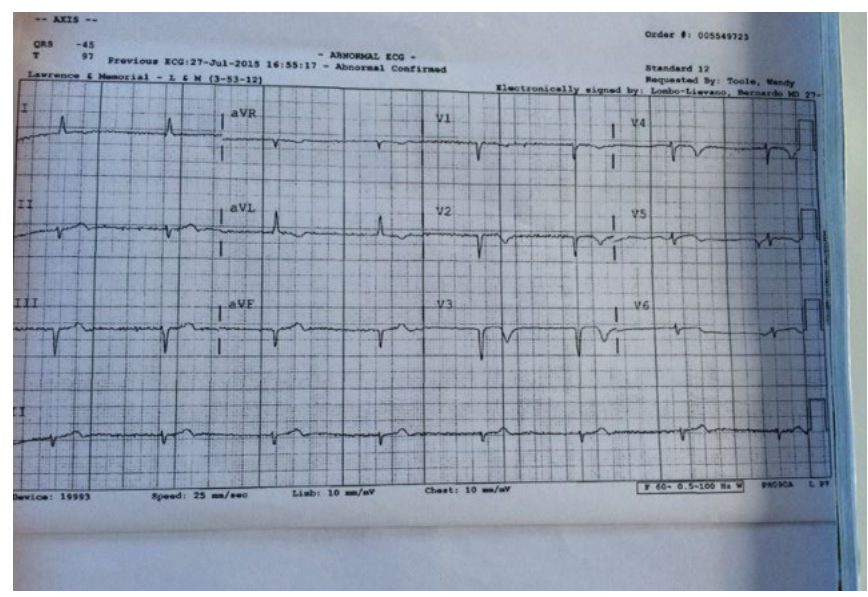

Figure 1 12-lead electrocardiogram demonstrating an episode of junctional escape bradycardia during initial presentation.

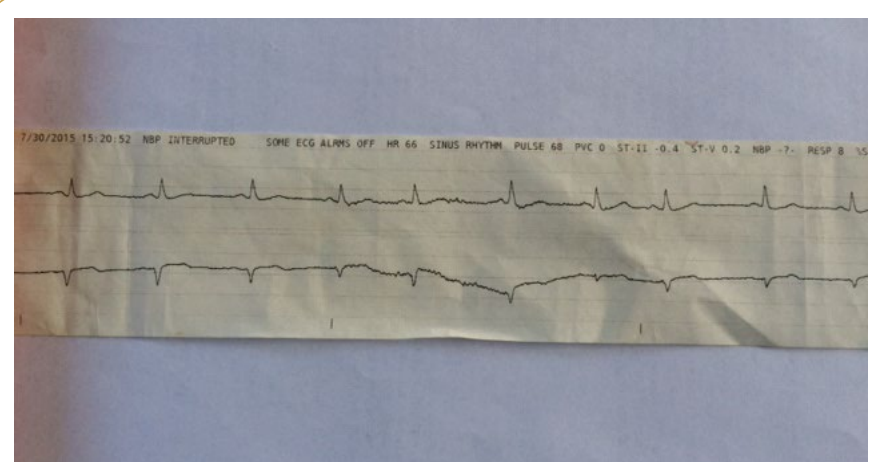

Figure 2

Rhythm strip demonstrating marked respiratory sinus arrhythmia. bradycardia and pauses of up to $4.4 \mathrm{~s}$. A fourth case was reported by Franzen et al. with second degree AV block and a pause of up to $9 \mathrm{~s}$. All authors conjectured that the mechanism of bradycardia or heart block was related to increased vagotonia associated with biliary inflammation. In our case, as the patient's biliary inflammation subsided and was treated with antibiotics, the arrhythmia ceased and the pacemaker was no longer needed. While we conjecture that the natural course of this illness is for vagotonia to wane as the inflammation wanes-our case and previous cases suggest as much-we cannot assert thus with great certainty.

Respiratory sinus arrhythmia is normal in young, healthy hearts and is believed to be modulated by vagotonic effects of inspiration. Recently, some have suggested an adaptive etiology relating to tightly matching ventilation and perfusion at various phases of respiration [5]. The diaphragms are richly innervated by the dorsal motor nucleus of the vagus nerve, as are other gastrointestinal visceral organs. It is not clear whether marked vagotonia associated with some cases of cholecystitis occurs due to inflammation of the gall bladder, right diaphragm, or both. While bradycardia, presumably due to increased vagotonia is known to occur (rarely) in other acute abdominal processes, this case is unique because it demonstrates two arrhythmiasabnormally slow junctional escape and respiratory-phasic variability in heart rate-that are typical of increased vagus effect and have not been previously reported with cholecystitis. In theory, atropine might be expected to reverse heart blocks and bradycardias induced by excess vagotonia [6]. Irrespective, the "cardio-biliary reflex" [3] is a rare epiphenomenon of acute cholecystitis and resolves with management/resolution of biliary inflammation. 


\section{References}

1 O'Reilly MV, Krauthamer MJ (1971) "Cope's sign" and reflex bradycardia in two patients with cholecystitis. Brit Med J 2: 146.

2 Lau YM, Hui WM, Lau CP (2015) Asystole complicating acalculous cholecystitis, the "Cope's sign" revisited. Int J Cardiol 182: 447-448.

3 Franzen D, Jung S, Fatio R, Brunckhorst CB (2009) Complete atrioventricular block in a patient with acute cholecystitis: A case of cardio-biliary reflex? Eur J Emerg Med 16: 346-347.
4 Krasna MJ, Flancbaum L (1986) Electrocardiographic changes in cardiac patients with acute gallbladder disease. Am Surg 52: 541-543.

5 Yasuma F, Hayano J (2004) Respiratory sinus arrhythmia: why does the heartbeat synchronize with respiratory rhythm? Chest 125: 683-690.

6 James TN, Urthaler F, Hageman GR (1980) Reflex heart block. Baroreflex, chemoreflex and bronchopulmonary reflex causes. Am J Cardiol 45: 1182-1188. 